\section{Rendimiento de los cuestionarios de sueño en la pesquisa de pacientes adultos con síndrome de apnea obstructiva del sueño según sexo}

\author{
FERNANDO SALDÍAS PEÑAFIEL ${ }^{1}$, GERARDO SALINAS ROSSEL ${ }^{a}$, \\ JOSEFINA CORTÉS MEZA ${ }^{\mathrm{b}}$, DANIELA FARÍAS NESVADBA ${ }^{\mathrm{b}}$, \\ ALEJANDRO PEÑALOZA TAPIA ${ }^{\mathrm{b}}$, CAROLINA AGUIRRE MARDONES ${ }^{2}$, \\ ORLANDO DÍAZ PATIÑO ${ }^{1}$
}

\section{Gender differences in clinical features and performance of sleep questionnaires in adults with obstructive sleep apnea syndrome}

Background:Women with obstructive sleep apnea-hypopnea syndrome (OSAS) are less likely to be assessed or to receive an appropriate diagnosis, and they may have poorer quality of life and survival rates. Aim: To assess gender-specific clinical differences in adult patients with OSAS. Material and Methods: A standardized clinical questionnaire and four sleep questionnaires (Berlin, Epworth Sleepiness Scale, STOP and STOP-Bang) were administered and anthropometric data were measured. Patients underwent an overnight in-laboratory polysomnography to confirm the diagnosis of OSAS. Receiver operating characteristic curves, sensitivity and specificity of clinical manifestations and sleep questionnaires were calculated. Results: Of 1,464 screened patients, 509 were female, $58.6 \%$ had moderate to severe OSAS. Clinical variables associated with OSAS risk in women were age, insomnia, nocturia, hypertension and cervical circumference. Paired by age and respiratory events, the snoring frequency was similar in both genders, although witnessed apneas and high cervical circumference and waist/hip ratio were more common in males. Morning headaches, insomnia, excessive daytime sleepiness, depression, anxiety and poor quality of sleep were more common in women. Women were older than men, more obese (although with an obesity pattern less centrally distributed) and referred hypertension, diabetes, depression and hypothyroidism with higher frequency. Sleep questionnaires performance were similar in both sexes. Conclusions: It is likely that women with OSAS may partially be underdiagnosed due to circumstances related to a different OSAS clinical expression.

(Rev Med Chile 2019; 147: 1291-1302)

Key words: Surveys and Questionnaires; Sleep Apnea, Obstructive; Sleep Apnea Syndromes.

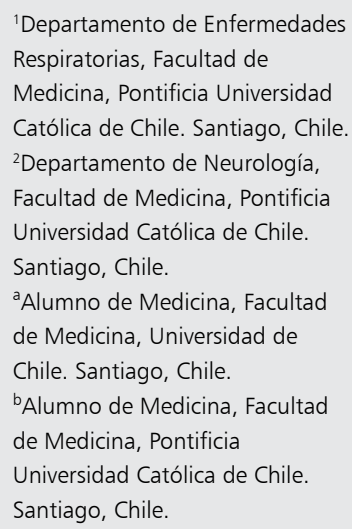

Trabajo no recibió

financiamiento.

Los autores declaran no tener conflictos de interés.

Recibido el 24 de marzo de 2019, aceptado el 12 de agosto de 2019.

\section{Correspondencia a :}

Dr. Fernando Saldías Peñafiel

Departamento de Enfermedades Respiratorias

División de Medicina, Facultad de Medicina. Pontificia Universidad Católica de Chile.

Diagonal Paraguay 362 - Sexto Piso. Santiago, Chile.

fsaldias@med.puc.cl
4 l síndrome de apnea-hipopnea obstructiva del sueño (SAHOS) es más prevalente en hombres en la edad media de la vida y en las mujeres después de la menopausia ${ }^{1-6}$. La diferencia en la prevalencia de SAHOS según sexo tiende a ser más pronunciada en los estudios realizados en las clínicas de sueño $0^{5,7}$. En los estudios de prevalencia de SAHOS poblacionales, definido por un índice de apnea-hipopnea $\geq 5$ eventos respiratorios/hora de sueño, se encontró una media de $22 \%$ (rango: 
9-37\%) en varones y 17\% (rango: $4-50 \%$ ) en mujeres ${ }^{8}$. Se ha planteado que las discrepancias observadas en la prevalencia de SAHOS según sexo dependen de las características de la población examinada y los criterios diagnósticos empleados en los diferentes estudios. Así, la prevalencia de SAHOS estimada en la cohorte de Wisconsin se basó inicialmente en el índice de apnea/hipopnea, siendo $24 \%$ en varones y $9 \%$ en mujeres; cuando los síntomas de somnolencia diurna se agregaron a los criterios diagnósticos, la prevalencia de SAHOS se redujo a $4 \%$ y $2 \%$, respectivamente ${ }^{1}$. En estudios más recientes realizados en la cohorte de Wisconsin se ha reportado una prevalencia de SAHOS de $14 \%$ en varones y $5 \%$ en mujeres ${ }^{6}$.

La menor prevalencia de SAHOS reportada en las mujeres, especialmente en las clínicas de sueño, puede deberse a diferencias en la notificación de los síntomas en hombres y mujeres. Es más probable que las mujeres refieran síntomas inespecíficos, tales como cefalea, fatigabilidad, trastornos del ánimo, ansiedad, insomnio o fragmentación del sueño $^{9-11}$. De hecho, cuando se controlan la edad, índice de masa corporal, gravedad de la enfermedad y la somnolencia diurna subjetiva, las mujeres reportan la presencia de insomnio y depresión con más frecuencia que los hombres ${ }^{12}$. A similar gravedad de la condición, los ronquidos estrepitosos, jadeo y las pausas respiratorias son reportados con menor frecuencia por las mujeres ${ }^{3}$. De este modo, las características clínicas distintivas de esta entidad podrían ser consideradas menos aceptables socialmente por las mujeres y, como resultado, no serían comunicadas en forma espontánea en la entrevista clínica. Los síntomas inespecíficos y la menor referencia de los síntomas clásicos podrían dificultar el diagnóstico clínico de SAHOS en mujeres, disminuyendo su pesquisa en los servicios de atención primaria y su derivación a los servicios de especialidad y clínicas de sueño ${ }^{1,2,13}$. En la mujer, las manifestaciones clínicas de SAHOS aumentan después de la menopausia y disminuyen con la terapia de reemplazo hormonal; además, las mujeres con SAHOS moderado-grave tienden a ser más obesas que los varones ${ }^{14}$. Es probable que los mecanismos fisiopatológicos determinantes de las manifestaciones clínicas de SAHOS difieran según el sexo ${ }^{15,16}$.

El propósito de este estudio es examinar las manifestaciones clínicas y el rendimiento de los cuestionarios de sueño en la pesquisa de SAHOS según el género en sujetos roncadores atendidos en una clínica de sueño.

\section{Pacientes y Métodos}

Estudio clínico prospectivo observacional que examinó las manifestaciones clínicas y el rendimiento de los cuestionarios de sueño en la pesquisa de SAHOS en pacientes adultos, de ambos sexos, atendidos en el Programa de Trastornos Respiratorios del Sueño de la Red de Salud UC Christus en el período de 1 de enero de 2015 hasta el 31 de diciembre de 2018. El protocolo fue revisado y aprobado por el Comité de Ética en Investigación de la Facultad de Medicina de la institución. Los pacientes respondieron a una entrevista clínica estandarizada de sueño, los cuestionarios de sueño autoadministrados (cuestionario de Berlín ${ }^{17}$ o cuestionario de calidad de sueño de Pittsburg ${ }^{18}$, STOP $^{19}$ y STOP-Bang ${ }^{20}$ ), índice de Flemons ${ }^{21}$, escala de somnolencia de Epworth ${ }^{22}$ y el inventario de depresión de Beck $^{23}$ o la escala de depresión y ansiedad, en el hospital ${ }^{24}$. A todos los pacientes se les realizó una polisomnografía (PSG) en el laboratorio de sueño para confirmar o descartar el diagnóstico de SAHOS. Se excluyeron del estudio a los pacientes: a) Menores de 18 años; b) Comorbilidades descompensadas; c) Diagnóstico previo de SAHOS; d) Enfermedades cardiovasculares, respiratorias, metabólicas o neurológicas crónicas en etapa avanzada; e) Incapacidad de responder a los cuestionarios clínicos o realizar el estudio de sueño.

En la evaluación clínica estandarizada realizada a los pacientes atendidos en el programa de trastornos respiratorios del sueño se consignaron la edad, sexo, ocupación, comorbilidades, uso de medicamentos, consumo de tabaco y alcohol, peso, talla, índice de masa corporal (IMC), perímetro cervical, circunferencia de cintura y cadera, historia de sueño: duración, presencia de ronquido, pausas respiratorias, insomnio, cefalea matinal, nicturia, inquietud psicomotora nocturna, sofocación nocturna, sueño poco reparador.

\section{Cuestionarios de sueño}

Cuestionario de Berlín. Permite estimar el riesgo de presentar trastornos respiratorios del sueño (SAHOS) en la población adulta atendida en los servicios de atención primaria ${ }^{17}$. Las preguntas del cuestionario se enfocaron en los siguientes aspec- 
tos: la presencia de ronquido, pausas respiratorias, somnolencia diurna, obesidad e hipertensión arterial. La primera categoría comprende cinco preguntas sobre el ronquido y apneas presenciadas. La segunda categoría comprende cuatro preguntas sobre la somnolencia diurna. La tercera categoría indaga acerca del antecedente de hipertensión arterial (PA $>140 / 90 \mathrm{mmHg}$ ) y obesidad (IMC $\left.>30 \mathrm{~kg} / \mathrm{m}^{2}\right)$. Un paciente tiene riesgo elevado de presentar SAHOS cuando posee dos o más categorías positivas.

Cuestionario de calidad de sueño de Pittsburgh. El cuestionario consta de 19 preguntas autoaplicadas que evalúan la calidad subjetiva del sueño, duración, latencia y eficiencia del sueño, trastornos del sueño, somnolencia diurna y el uso de fármacos hipnóticos ${ }^{18}$.

Cuestionarios STOP y STOP-Bang. El cuestionario STOP consiste en cuatro preguntas que evalúan la presencia de ronquido intenso, somnolencia diurna o fatigabilidad, apneas presenciadas durante el sueño e hipertensión arterial ${ }^{19}$. En el modelo extendido, STOP-Bang, se incorporan cuatro parámetros adicionales: edad, género, índice de masa corporal y circunferencia cervical ${ }^{20}$. Las respuestas a las preguntas de ambos cuestionarios son dicotómicas ( $\mathrm{Si} / \mathrm{No}$ ) y las puntuaciones fluctúan entre 0 y 4 u 8 puntos, respectivamente. Se identifica a los sujetos con riesgo elevado de SAHOS cuando se responden afirmativamente al menos dos (STOP) o tres (STOP-Bang) preguntas $^{19,20,25}$.

Escala de somnolencia de Epworth. Cuestionario autoadministrado que solicita a los pacientes que califiquen la probabilidad de quedarse dormido en ocho situaciones o actividades específicas de la vida cotidiana ${ }^{22}$. Se califica en una escala de 0 a 3 puntos cada situación y la puntuación puede oscilar entre 0 y 24. La puntuación de la escala de Epworth se clasifica de bajo riesgo de somnolencia (menor de 11 puntos) y alto riesgo de somnolencia (11-24 puntos).

\section{Estudio trastornos respiratorios del sueño}

El diagnóstico de los trastornos respiratorios del sueño se realizó con un equipo de polisomnografía Alice 5 (Royal Philips, Respironics, Eindhoven, Países Bajos) en el laboratorio de sueño analizados según las normas del Manual de Etapificación de Polisomnografía de Reschtscha- ffen y Kales ${ }^{26}$. El análisis de la PSG fue realizado manualmente por un médico especialista en trastornos respiratorios del sueño que desconocía los antecedentes clínicos del paciente y los resultados de los cuestionarios de sueño; consignando la duración del estudio, etapas del sueño y microdespertares, posición corporal, número de apneas e hipopneas obstructivas, centrales y mixtas, caída de la saturación arterial de oxígeno bajo 90\% (CT90\%) y número de episodios de desaturación mayor de 4\%. Se calculó el número de eventos respiratorios (IAH) dividiendo el número total de apneas e hipopneas por la duración del sueño. La ejecución e interpretación de los exámenes siguió las recomendaciones de la Academia Americana de Medicina del Sueño ${ }^{27}$. El diagnóstico y la gravedad del SAHOS se clasificó siguiendo las recomendaciones internacionales: registro normal (IAH $<5$ eventos/h), SAHOS leve (IAH: 5-14,9), moderado (IAH: 15-29,9) y grave $(\mathrm{IAH} \geq 30$ eventos/h).

\section{Análisis estadístico}

Los resultados fueron expresados como valores promedio \pm desviación estándar para las variables numéricas y en porcentaje para las medidas en escala nominal. Las variables cualitativas fueron comparadas mediante la prueba de chi cuadrado y el test exacto de Fisher, y las variables continuas según su distribución con la prueba t de Student o la prueba de Mann-Whitney. Para ello se utilizó el programa estadístico SPSS 25.0 (SPSS Inc, IBM Company, Chicago, IL, USA). Las variables clínicas y reglas predictivas asociadas al riesgo de SAHOS fueron examinadas en 509 mujeres pareadas por edad ( \pm 3 años) y gravedad de las condición (IAH \pm 5 eventos respiratorios/h) con 509 varones de la cohorte examinada. Se midió la sensibilidad, especificidad, valores predictivos y las razones de probabilidades (odds ratio) de los índices predictores de SAHOS, considerando los puntos de corte definidos por los autores ${ }^{17-22}$. Para comparar la sensibilidad y especificidad de las distintas reglas predictoras se utilizó el test de $\mathrm{McNemar}$. Las curvas receptor operador (ROC) de los modelos predictivos nos permitió comparar su capacidad para predecir el diagnóstico de SAHOS. Para ello, se consignó el área bajo la curva ROC, el intervalo de confianza de $95 \%$ y valor de p. Las diferencias entre las variables fueron consideradas significativas con un valor de $\mathrm{p}<0,05$. 


\section{Resultados}

Se evaluaron 1.464 pacientes adultos en el programa de trastornos respiratorios del sueño, 509 eran mujeres, 90\% tenía comorbilidades, especialmente hipertensión arterial, diabetes mellitus, dislipidemia, reflujo gastroesofágico y trastornos del ánimo. Las principales manifes- taciones clínicas eran ronquido intenso, apneas presenciadas por testigos, somnolencia diurna, sueño poco reparador, nicturia, cefalea matinal, boca seca al despertar, disminución de la memoria; $82,7 \%$ de los pacientes tenía SAHOS (IAH mayor de 5 eventos/h de sueño), siendo 58,6\% SAHOS moderado-grave (Tabla 1).

Las mujeres con SAHOS predominaron entre

Tabla 1. Características clínicas de los pacientes atendidos en el programa de trastornos respiratorios del sueño

\begin{tabular}{|c|c|c|}
\hline Características & $n(\bar{x} \pm D E)$ & Porcentaje (rango) \\
\hline$n$ & 1.464 & \\
\hline Edad (años) & $54,4 \pm 15,1$ & $18-92$ \\
\hline Género (masculino-femenino) & $955-509$ & $65,2-34,8$ \\
\hline Consumo de tabaco & $568 / 1.461$ & 38,9 \\
\hline Comorbilidades & 1.315 & 89,8 \\
\hline Enfermedad cardiovascular & 169 & 11,5 \\
\hline Hipertensión arterial & 728 & 49,7 \\
\hline Diabetes mellitus & 322 & 22,0 \\
\hline Dislipidemia & 443 & 30,3 \\
\hline Rinitis alérgica & 358 & 24,4 \\
\hline Asma-EPOC & $130-69$ & $8,9-4,7$ \\
\hline Reflujo gastroesofágico & 533 & 36,4 \\
\hline Depresión & 387 & 26,4 \\
\hline Hipotiroidismo & 243 & 16,6 \\
\hline Peso (Kg) & $85,8 \pm 17,6$ & $32-200$ \\
\hline Talla (cm) & $167,5 \pm 9,5$ & $130-195$ \\
\hline $\mathrm{IMC}\left(\mathrm{Kg} / \mathrm{m}^{2}\right)$ & $30,6 \pm 5,6$ & $16,7-58,5$ \\
\hline Perímetro cervical (cm) & $41,6 \pm 4,4$ & $27-58$ \\
\hline Circunferencia de cintura $(\mathrm{cm})$ & $108,1 \pm 13,6$ & $61-166$ \\
\hline Circunferencia de cadera $(\mathrm{cm})$ & $109,8 \pm 10,9$ & $83-166$ \\
\hline Índice cintura/cadera & $0,98 \pm 0,08$ & $0,58-1,41$ \\
\hline Presión arterial sistólica (mmHg) & $126,9 \pm 19,8$ & $81-230$ \\
\hline Presión arterial diastólica $(\mathrm{mmHg})$ & $80,1 \pm 13,1$ & $50-152$ \\
\hline Roncador habitual & 1.131 & 77,3 \\
\hline Apneas presenciadas & 1.035 & 70,7 \\
\hline Somnolencia diurna excesiva & 690 & 47,1 \\
\hline Sueño poco reparador & 1.016 & 69,4 \\
\hline Insomnio & 679 & 46,4 \\
\hline Cefalea matinal & 610 & 41,7 \\
\hline Boca seca al despertar & $315 / 414$ & 76,1 \\
\hline Nicturia & 907 & 62,0 \\
\hline Sofocación nocturna & $328 / 730$ & 44,9 \\
\hline Inquietud psicomotora nocturna & $381 / 799$ & 47,7 \\
\hline Disminución de la memoria & $324 / 414$ & 78,2 \\
\hline SAHOS leve-moderado-grave & $353-320-538$ & $24,1-21,9-36,7$ \\
\hline
\end{tabular}

Nota: $\overline{\mathrm{X}} \pm \mathrm{DE}$ : Promedio \pm Desviación estándar; EPOC: Enfermedad pulmonar obstructiva crónica; IMC: Índice de masa corporal; SAHOS: Síndrome de apnea-hipopnea obstructiva del sueño. 
Síndrome de apnea obstructiva de sueño según género - F. Saldías et al

la cuarta y séptima década de la vida (Figura 1), tenían más comorbilidades, especialmente hipertensión arterial, diabetes mellitus, depresión e hipotiroidismo comparado con los varones.
El reporte de apneas presenciadas por testigos predominó en varones; mientras que la cefalea matinal, insomnio y sueño poco reparador fueron más prevalentes en las mujeres (Tabla 2).

Tabla 2. Características clínicas de los pacientes atendidos en el programa de trastornos respiratorios del sueño según sexo

\begin{tabular}{|c|c|c|c|}
\hline Características & Hombres & Mujeres & $\mathbf{p}$ \\
\hline$n$ & 955 & 509 & \\
\hline Edad (años) & $53,3 \pm 14,6$ & $56,4 \pm 15,6$ & 0,001 \\
\hline Consumo de tabaco & $402 / 952(42,2 \%)$ & $166 / 509(32,6 \%)$ & 0,001 \\
\hline Comorbilidades & $841(88,1 \%)$ & $474(93,1 \%)$ & 0,002 \\
\hline Enfermedad cardiovascular & $125(13,1 \%)$ & $44(8,6 \%)$ & 0,011 \\
\hline Hipertensión arterial & $453(47,4 \%)$ & $275(54,0 \%)$ & 0,017 \\
\hline Diabetes mellitus & $191(20,0 \%)$ & $131(25,7 \%)$ & 0,012 \\
\hline Dislipidemia & $279(29,2 \%)$ & $164(32,2 \%)$ & 0,563 \\
\hline Rinitis alérgica & $229(24,0 \%)$ & $129(25,3 \%)$ & 0,171 \\
\hline Asma-EPOC & $73(7,6 \%)-46(4,8 \%)$ & $57(11,1 \%)-23(4,5 \%)$ & $0,03-0,79$ \\
\hline Reflujo gastroesofágico & $332(34,8 \%)$ & $201(39,5 \%)$ & 0,074 \\
\hline Depresión & $175(18,3 \%)$ & $212(41,7 \%)$ & 0,001 \\
\hline Hipotiroidismo & $76(8,0 \%)$ & $167(32,8 \%)$ & 0,001 \\
\hline Peso (Kg) & $90,5 \pm 16,6$ & $76,9 \pm 15,8$ & 0,001 \\
\hline Talla $(\mathrm{cm})$ & $172,5 \pm 6,6$ & $158,0 \pm 6,6$ & 0,001 \\
\hline $\mathrm{IMC}\left(\mathrm{Kg} / \mathrm{m}^{2}\right)$ & $30,4 \pm 5,1$ & $30,9 \pm 6,5$ & 0,106 \\
\hline Perímetro cervical (cm) & $43,2 \pm 3,6$ & $38,2 \pm 4,2$ & 0,001 \\
\hline Circunferencia de cintura $(\mathrm{cm})$ & $109,6 \pm 12,7$ & $104,9 \pm 14,8$ & 0,001 \\
\hline Circunferencia de cadera $(\mathrm{cm})$ & $108,9 \pm 9,7$ & $112,0 \pm 13,1$ & 0,001 \\
\hline Índice cintura/cadera & $1,01 \pm 0,07$ & $0,93 \pm 0,07$ & 0,001 \\
\hline Presión arterial sistólica (mmHg) & $126,7 \pm 19,7$ & $127,3 \pm 20,1$ & 0,581 \\
\hline Presión arterial diastólica $(\mathrm{mmHg})$ & $81,1 \pm 13,2$ & $78,0 \pm 12,7$ & 0,001 \\
\hline Roncador habitual & $758(79,4 \%)$ & $373(73,3 \%)$ & 0,008 \\
\hline Apneas presenciadas & $728(76,2 \%)$ & $307(60,3 \%)$ & 0,001 \\
\hline Somnolencia diurna excesiva & $439(46,0 \%)$ & $251(49,3 \%)$ & 0,223 \\
\hline Sueño poco reparador & $627(65,7 \%)$ & $389(76,4 \%)$ & 0,001 \\
\hline Insomnio & $379(39,7 \%)$ & $300(58,9 \%)$ & 0,001 \\
\hline Cefalea matinal & $322(33,7 \%)$ & $288(56,6 \%)$ & 0,001 \\
\hline Boca seca al despertar & $174 / 237(73,4 \%)$ & $141 / 177(79,7 \%)$ & 0,141 \\
\hline Nicturia & $577(60,4 \%)$ & $330(64,8 \%)$ & 0,097 \\
\hline Sofocación nocturna & $202 / 440(45,9 \%)$ & $126 / 290(43,4 \%)$ & 0,514 \\
\hline Inquietud psicomotora nocturna & $241 / 490(49,2 \%)$ & $140 / 309(45,3 \%)$ & 0,286 \\
\hline Disminución de la memoria & $179 / 237(75,5 \%)$ & 145/177 (81,9\%) & 0,119 \\
\hline SAHOS leve-moderado-grave & $209-210-419$ & $144-110-119$ & 0,001 \\
\hline
\end{tabular}

Nota: EPOC: Enfermedad pulmonar obstructiva crónica; IMC: Índice de masa corporal; SAHOS: Síndrome de apnea-hipopnea obstructiva del sueño. Los valores son expresados como promedio \pm desviación estándar o número y porcentajes. 

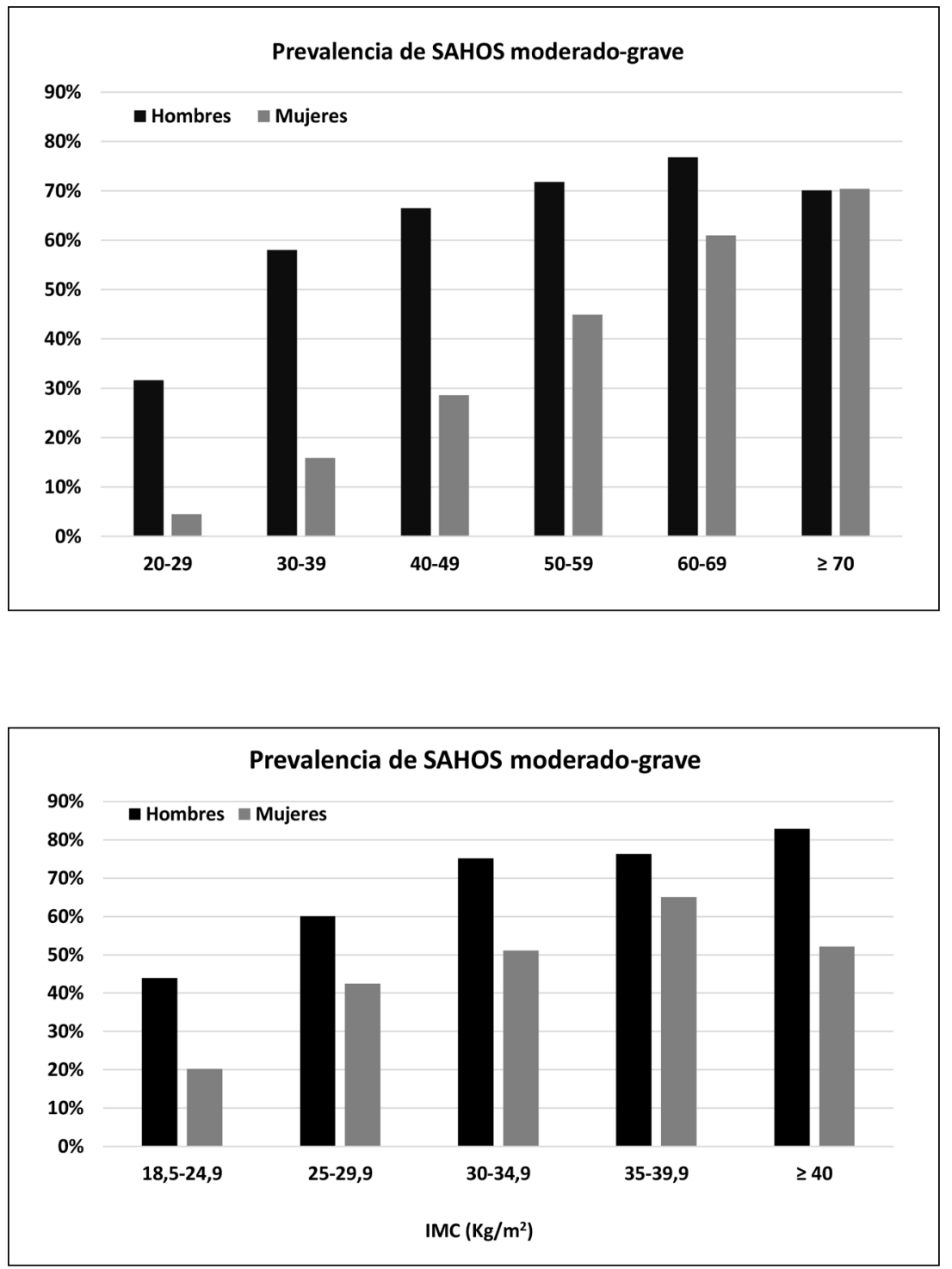

Figura 1. Distribución etárea de los pacientes adultos con SAHOS moderado-grave atendidos en el programa de trastornos respiratorios del sueño.
Figura 2. Prevalencia de SAHOS moderado-grave según estado nutricional en pacientes adultos atendidos en el programa de trastornos respiratorios del sueño.
Las mujeres con trastornos respiratorios del sueño, pareadas por edad y gravedad de la condición con los varones, eran más obesas y reportaban síntomas de ansiedad, depresión, somnolencia diurna excesiva y pobre calidad del sueño con mayor frecuencia comparado con los varones. En ambos sexos, el riesgo de SAHOS aumentó significativamente en sujetos con sobrepeso y obesidad (Figura 2). La historia de ronquido habitual, $\mathrm{SaO}_{2}$ basal, $\mathrm{SaO}_{2}$ mínima e índice de desaturación del $4 \%$ fueron similares en ambos sexos; mientras que en los varones fue más frecuente la referencia de apneas presenciadas por testigos y el perímetro cervical e índice cintura/cadera eran más elevados (Tablas 3 y 4 ).

En el análisis multivariado, los principales predictores de riesgo de SAHOS en varones fueron la edad, apneas presenciadas, somnolencia diurna, insomnio, nicturia y perímetro cervical; mientras que en las mujeres, los predictores independientes de SAHOS fueron la edad, insomnio, nicturia, hipertensión arterial y perímetro cervical (Tabla 5). 
Tabla 3. Factores de riesgo y cuestionarios de sueño de los pacientes atendidos en el programa de trastornos respiratorios del sueño según sexo

\begin{tabular}{|c|c|c|c|}
\hline Características & Hombres & Mujeres & $\mathbf{p}$ \\
\hline $\mathrm{n}$ & 955 & 509 & \\
\hline Duración del sueño (horas) & $7,04 \pm 1,59$ & $7,26 \pm 1,87$ & 0,018 \\
\hline $\mathrm{IMC}>30 \mathrm{~kg} / \mathrm{m}^{2}$ & $441 / 926(47,6 \%)$ & $238 / 485(49,1 \%)$ & 0,211 \\
\hline Perímetro cervical $>40 \mathrm{~cm}$ & $530 / 666(79,6 \%)$ & $81 / 305(26,6 \%)$ & 0,001 \\
\hline Índice cintura/cadera $>0,94$ & $545 / 630(86,5 \%)$ & $133 / 281(47,3 \%)$ & 0,001 \\
\hline $\begin{array}{l}\text { Cuestionario de depresión de Beck sugerente } \\
\text { depresión moderada-grave }\end{array}$ & $72 / 695(10,3 \%)$ & $86 / 318(27,0 \%)$ & 0,001 \\
\hline HADS-ansiedad & $7,1 \pm 4,4$ & $8,5 \pm 4,6$ & 0,001 \\
\hline HADS-depresión & $5,1 \pm 4,0$ & $5,9 \pm 4,2$ & 0,001 \\
\hline Score PSQI & $8,8 \pm 3,9$ & $10,5 \pm 4,1$ & 0,001 \\
\hline Cuestionario de Berlín: Alto riesgo de SAHOS & $251 / 431(58,2 \%)$ & $151 / 288(52,4 \%)$ & 0,057 \\
\hline STOP: Alto riesgo de SAHOS & $804 / 955(84,2 \%)$ & $385 / 509(75,6 \%)$ & 0,001 \\
\hline STOP-Bang: Alto riesgo de SAHOS & $700 / 718(97,5 \%)$ & $267 / 329(81,1 \%)$ & 0,001 \\
\hline Escala de Epworth & $10,7 \pm 5,7$ & $11,0 \pm 5,7$ & 0,338 \\
\hline Escala de Epworth: Somnolencia diurna excesiva & $437 / 937(46,6 \%)$ & $256 / 491(52,1 \%)$ & 0,049 \\
\hline Eficiencia del sueño (\%) & $77,5 \pm 14,9$ & $78,0 \pm 13,2$ & 0,526 \\
\hline $\mathrm{SaO}_{2}$ basal (\%) & $96,1 \pm 1,9$ & $95,9 \pm 2,4$ & 0,009 \\
\hline $\mathrm{SaO}_{2}$ media (\%) & $92,3 \pm 3,4$ & $92,1 \pm 4,1$ & 0,320 \\
\hline $\mathrm{SaO}_{2}$ mínima (\%) & $79,9 \pm 9,3$ & $80,9 \pm 9,7$ & 0,054 \\
\hline CT-90\% (\%) & $14,1 \pm 21,5$ & $15,8 \pm 26,9$ & 0,188 \\
\hline ID4\% & $26,8 \pm 22,2$ & $21,0 \pm 22,1$ & 0,001 \\
\hline Frecuencia cardiaca media (lat/min) & $64,8 \pm 9,5$ & $68,4 \pm 9,8$ & 0,001 \\
\hline Índice de apnea-hipopnea & $30,4 \pm 24,3$ & $19,3 \pm 20,5$ & 0,001 \\
\hline
\end{tabular}

Nota: IMC: Índice de masa corporal; HADS: Hospital Anxiety and Depression Scale; PSQI: Pittsburgh Sleep Quality Index; SAHOS: Síndrome de apnea-hipopnea obstructiva del sueño; $\mathrm{SaO}_{2}$ : Saturación arterial de oxígeno; CT90\%: Porcentaje del tiempo con $\mathrm{SaO}_{2}$ bajo 90\%; ID4\%: Índice de desaturación del 4\%. Los valores son expresados como promedio \pm desviación estándar o número y porcentajes.

La historia de ronquido intenso habitual, pausas respiratorias durante el sueño, somnolencia diurna e hipertensión arterial fue similar en sujetos de ambos sexos con SAHOS moderado-grave. El índice de Flemons y cuestionario STOP-Bang tuvieron discreto mejor desempeño en la pesquisa de SAHOS en las mujeres; las variables antropométricas y el cuestionario de Berlín tuvieron similar desempeño en ambos sexos; mientras que la escala de Epworth se asoció al riesgo de SAHOS solo en los varones (Tabla 6). En ambos sexos, los cuestionarios de sueño STOP y STOP-Bang fueron más sensibles y el índice de Flemons fue más específico en la pesquisa de pacientes con SAHOS moderado-grave (Tabla 7).

\section{Discusión}

Los principales hallazgos del estudio fueron: a) Un tercio de los pacientes atendidos en la clínica de sueño con trastornos respiratorios del sueño 
Tabla 4. Evaluación del desempeño de los predictores clínicos de SAHOS según sexo en pacientes adultos atendidos en el programa de trastornos respiratorios del sueño pareados por edad y gravedad de la enfermedad

\begin{tabular}{|c|c|c|c|}
\hline Características & Hombres & Mujeres & $\mathbf{p}$ \\
\hline$n$ & 509 & 509 & \\
\hline Edad (años) & $56,7 \pm 15,5$ & $55,6 \pm 15,9$ & 0,264 \\
\hline Duración del sueño (horas) & $7,04 \pm 1,61$ & $7,26 \pm 1,87$ & 0,045 \\
\hline Roncador habitual & $398 / 509(78,2 \%)$ & $379 / 509(74,5 \%)$ & 0,162 \\
\hline Apneas presenciadas & $368 / 509$ (72,3\%) & $304 / 509(59,7 \%)$ & 0,001 \\
\hline $\mathrm{IMC}>30 \mathrm{~kg} / \mathrm{m}^{2}$ & $193 / 490(39,4 \%)$ & $238 / 485(49,1 \%)$ & 0,002 \\
\hline Perímetro cervical $>40 \mathrm{~cm}$ & 246/332 (74,1\%) & $81 / 305(26,6 \%)$ & 0,001 \\
\hline Índice cintura/cadera $>0,94$ & 281/327 (85,9\%) & $133 / 281(47,3 \%)$ & 0,001 \\
\hline $\begin{array}{l}\text { Cuestionario de depresión de Beck sugerente } \\
\text { depresión moderada-grave }\end{array}$ & 40/352 (11,4\%) & $86 / 318(27,0 \%)$ & 0,001 \\
\hline HADS-ansiedad & $7,1 \pm 4,6$ & $8,5 \pm 4,6$ & 0,001 \\
\hline HADS-depresión & $4,9 \pm 3,9$ & $5,9 \pm 4,2$ & 0,001 \\
\hline Score PSQI & $8,8 \pm 4,0$ & $10,5 \pm 4,1$ & 0,001 \\
\hline Cuestionario de Berlín: Alto riesgo de SAHOS & $150 / 262(57,3 \%)$ & $151 / 288(52,4 \%)$ & 0,257 \\
\hline STOP: Alto riesgo de SAHOS & $413 / 509(81,1 \%)$ & $385 / 509(75,6 \%)$ & 0,034 \\
\hline STOP-Bang: Alto riesgo de SAHOS & $354 / 363(97,5 \%)$ & $267 / 329(81,1 \%)$ & 0,001 \\
\hline Escala de Epworth & $9,9 \pm 5,5$ & $11,0 \pm 5,7$ & 0,002 \\
\hline Escala de Epworth: Somnolencia diurna excesiva & $200 / 497(40,2 \%)$ & $256 / 491(52,1 \%)$ & 0,001 \\
\hline $\mathrm{SaO}_{2}$ basal (\%) & $96,1 \pm 1,9$ & $95,9 \pm 2,4$ & 0,141 \\
\hline $\mathrm{SaO}_{2}$ media (\%) & $92,8 \pm 2,8$ & $92,1 \pm 4,1$ & 0,002 \\
\hline $\mathrm{SaO}_{2}$ mínima (\%) & $81,8 \pm 8,1$ & $80,9 \pm 9,7$ & 0,109 \\
\hline CT-90\% (\%) & $11,3 \pm 20,2$ & $15,8 \pm 26,9$ & 0,003 \\
\hline ID4\% & $18,5 \pm 18,4$ & $21,0 \pm 22,1$ & 0,051 \\
\hline Frecuencia cardiaca media (lat/min) & $63,6 \pm 9,4$ & $68,4 \pm 9,8$ & 0,001 \\
\hline Índice de apneas-hipopneas & $20,5 \pm 20,6$ & $19,3 \pm 20,5$ & 0,352 \\
\hline
\end{tabular}

Nota: IMC: Índice de masa corporal; HADS: Hospital Anxiety and Depression Scale; PSQI: Pittsburgh Sleep Quality Index; SAHOS: Síndrome de apnea-hipopnea obstructiva del sueño; $\mathrm{SaO}_{2}$ : Saturación arterial de oxígeno; CT90\%: Porcentaje del tiempo con $\mathrm{SaO}_{2}$ bajo 90\%; ID4\%: Índice de desaturación del 4\%. Los valores son expresados como promedio \pm desviación estándar o número y porcentajes.

eran mujeres; b) La prevalencia de SAHOS moderado-grave en las mujeres se eleva progresivamente entre la cuarta y séptima década de la vida; c) La historia de ronquido habitual o apneas presenciadas fue menos reportada en mujeres; d) La historia de somnolencia diurna, insomnio, cefalea matinal y sueño poco reparador fue más prevalente en las mujeres; e) Las mujeres con SAHOS reportan síntomas de ansiedad, depresión y pobre calidad del sueño con mayor frecuencia comparado con los varones; $\mathrm{f}$ ) Las variables clínicas asociadas al riesgo de SAHOS en mujeres fueron la edad, historia de insomnio, nicturia, hipertensión arterial y perímetro cervical; g) El índice de Flemons y el cuestionario STOP-Bang tuvieron mejor desempeño en la pesquisa de SAHOS en mujeres; mientras que la escala de Epworth tuvo mejor desempeño en varones. 
Tabla 5. Variables clínicas asociadas al riesgo de síndrome de apneas obstructivas del sueño de magnitud moderada-grave según sexo. Análisis multivariado

\begin{tabular}{|lccccc|}
\hline Riesgo de SAHOS & Coeficiente & Error estándar & Odds ratio & IC95\% & p \\
Hombres & & & & & \\
Edad & 0,0169 & 0,0068 & 1,02 & $1,01-1,03$ & 0,0141 \\
Apneas & 0,7379 & 0,2312 & 2,09 & $1,33-3,29$ & 0,0014 \\
Somnolencia & 0,3853 & 0,2085 & 1,47 & $1,01-2,21$ & 0,0499 \\
Insomnio & $-0,5178$ & 0,1945 & 0,59 & $0,41-0,87$ & 0,0078 \\
Nicturia & 0,4219 & 0,2039 & 1,53 & $1,02-2,27$ & 0,0385 \\
Perímetro cervical & 0,1591 & 0,0297 & 1,17 & $1,11-1,24$ & 0,0001 \\
Mujeres & & & & & 0,0179 \\
Edad & 0,0293 & 0,0123 & 1,03 & $1,01-1,06$ & 0,0324 \\
Insomnio & $-0,6318$ & 0,2953 & 0,53 & $0,30-0,94$ & 0,03 \\
Hipertensión & 0,8198 & 0,3399 & 2,27 & $1,17-4,42$ & 0,0159 \\
Nicturia & 0,8710 & 0,3754 & 2,39 & $1,14-4,98$ & 0,0203 \\
Perímetro cervical & 0,1808 & 0,0436 & 1,20 & $1,10-1,31$ & 0,0001 \\
\hline
\end{tabular}

Tabla 6. Evaluación del área bajo la curva receptor operador (AUC) de los predictores clínicos y cuestionarios de sueño en el diagnóstico de trastornos respiratorios del sueño (SAHOS moderado-grave)

\begin{tabular}{|lll|}
\hline Predictores clínicos & $\begin{array}{l}\text { Hombres } \\
\text { AUC (IC95\%, p) }\end{array}$ & $\begin{array}{l}\text { Mujeres } \\
\text { AUC (IC95\%, p) }\end{array}$ \\
\hline Edad* & $0,59(0,56-0,62, p<0,0001)$ & $0,72(0,68-0,76, p<0,0001)$ \\
\hline Ronquido-apnea-SDE & $0,58(0,55-0,62, p<0,0001)$ & $0,54(0,49-0,58, p: 0,0610)$ \\
\hline Índice de masa corporal & $0,66(0,62-0,69, p<0,0001)$ & $0,66(0,61-0,70, p<0,0001)$ \\
\hline Perímetro cervical & $0,65(0,62-0,69, p<0,0001)$ & $0,70(0,65-0,75, p<0,0001)$ \\
\hline Índice cintura/cadera & $0,58(0,54-0,62, p: 0,0005)$ & $0,55(0,48-0,60, p: 0,2032)$ \\
\hline Índice de Flemons ${ }^{+}$ & $0,64(0,60-0,67, p<0,0001)$ & $0,72(0,67-0,77, p<0,0001)$ \\
\hline Cuestionario de Berlín & $0,57(0,52-0,61, p: 0,0066)$ & $0,56(0,50-0,62, p: 0,0296)$ \\
\hline STOP & $0,62(0,59-0,65, p<0,0001)$ & $0,67(0,63-0,71, p<0,0001)$ \\
\hline STOP-Bang ${ }^{+}$ & $0,64(0,60-0,67, p<0,0001)$ & $0,72(0,66-0,76, p<0,0001)$ \\
\hline Escala de Epworth* & $0,60(0,57-0,64, p<0,0001)$ & $0,50(0,46-0,55, p: 0,8979)$ \\
\hline
\end{tabular}

Nota: AUC: Área bajo la curva receptor operador, IC95\%: Intervalo de confianza de 95\%. SDE: Somnolencia diurna excesiva. ${ }^{+} p<0,05 ;{ }^{*} p<0,001$.

En nuestro estudio, la historia clásica de ronquido intenso habitual y apneas presenciadas por testigos fue más prevalente en varones, mientras que los síntomas inespecíficos de ansiedad, depresión, insomnio, cefalea matinal, somnolencia diurna y pobre calidad del sueño fueron reportados con mayor frecuencia por las mujeres ${ }^{3,31}$. En general, las mujeres diagnosticadas con SAHOS tienen mayor edad y menor gravedad de la con- dición que los varones (Tabla 2$)^{32,33}$. En la Tabla 8 se mencionan las principales diferencias en las manifestaciones clínicas de SAHOS según sexo descritas en la literatura. De hecho, se ha planteado que la ausencia de los síntomas clásicos y la presencia de síntomas inespecíficos en la mujer dificultaría y retrasaría el diagnóstico de los trastornos respiratorios del sueño en los servicios de atención primaria ${ }^{3,11}$. 
Tabla 7. Sensibilidad, especificidad, valores predictivos y odds ratio de las categorías de alto riesgo de los cuestionarios de sueño en el diagnóstico de trastornos respiratorios del sueño (SAHOS moderado-grave)

\begin{tabular}{|lccccrrr|}
\hline Cuestionarios & Sensibilidad Especificidad & VPP & VPN & Odds ratio & IC95\% & p \\
Hombres & & & & & & & \\
$\quad$ Índice de Flemons & 0,51 & 0,67 & 0,73 & 0,43 & 2,05 & $1,48-2,82$ & $<0,0001$ \\
Berlín & 0,66 & 0,47 & 0,64 & 0,49 & 1,72 & $1,16-2,55$ & 0,0067 \\
STOP & 0,89 & 0,25 & 0,70 & 0,55 & 2,82 & $1,98-4,02$ & $<0,0001$ \\
STOP-Bang & 0,99 & 0,05 & 0,65 & 0,67 & 3,79 & $1,40-10,2$ & 0,0086 \\
Epworth & 0,51 & 0,62 & 0,72 & 0,40 & 1,65 & $1,26-2,18$ & 0,0003 \\
Mujeres & & & & & & & \\
$\quad$ Índice de Flemons & 0,26 & 0,89 & 0,63 & 0,62 & 2,77 & $1,53-5,00$ & 0,0008 \\
Berlín & 0,63 & 0,50 & 0,48 & 0,64 & 1,69 & $1,05-2,73$ & 0,0318 \\
STOP & 0,88 & 0,32 & 0,51 & 0,76 & 3,40 & $2,13-5,43$ & $<0,0001$ \\
STOP-Bang & 0,94 & 0,29 & 0,51 & 0,86 & 6,32 & $3,00-13,3$ & $<0,0001$ \\
\hline
\end{tabular}

Nota: VPP: Valor predictivo positivo, VPN: Valor predictivo negativo, IC95\%: Intervalo de confianza de $95 \%$.

Tabla 8. Diferencias de género en los síntomas de presentación de los pacientes adultos con síndrome de apnea obstructiva del sueño

\begin{tabular}{|c|c|c|}
\hline Estudio & Muestra & $\begin{array}{l}\text { Características clínicas del SAHOS en mujeres comparado con } \\
\text { los varones }\end{array}$ \\
\hline $\begin{array}{l}\text { Ambrogetti et al. }{ }^{31} \\
1991\end{array}$ & $22 \mathrm{~F}-44 \mathrm{M}$ & $\begin{array}{l}\text { Predominio de fatiga matinal, cefalea matinal, dificultad para iniciar el sueño } \\
\text { Reporte de somnolencia diurna similar en ambos sexos } \\
\text { Menor reporte de síntomas clásicos y apneas presenciadas por testigos }\end{array}$ \\
\hline $\begin{array}{l}\text { Redline et al. }{ }^{3} \\
1994\end{array}$ & 389 sujetos & Menor reporte de síntomas clásicos y apneas presenciadas por testigos \\
\hline $\begin{array}{l}\text { Guilleminault et al. }{ }^{38} \\
1995\end{array}$ & $334 \mathrm{~F}-100 \mathrm{M}$ & Mayor retraso en el diagnóstico \\
\hline $\begin{array}{l}\text { Young et al. }{ }^{2} \\
1996\end{array}$ & $388 \mathrm{~F}-551 \mathrm{M}$ & Los síntomas reportados fueron similares en ambos sexos \\
\hline $\begin{array}{l}\text { Pillar et al. }{ }^{9} \\
1998\end{array}$ & $294 \mathrm{~F}-1.977 \mathrm{M}$ & Los síntomas de ansiedad y depresión fueron más reportados por mujeres \\
\hline $\begin{array}{l}\text { Chervin et al. }{ }^{39} \\
2000\end{array}$ & $73 \mathrm{~F}-117 \mathrm{M}$ & Predominio de fatiga, cansancio y falta de energía \\
\hline $\begin{array}{l}\text { Walker et al. }{ }^{40} \\
2001\end{array}$ & $111 \mathrm{~F}-575 \mathrm{M}$ & Los síntomas reportados fueron similares en ambos sexos \\
\hline $\begin{array}{l}\text { Resta et al. }{ }^{41} \\
2003\end{array}$ & $148 \mathrm{~F}-82 \mathrm{M}$ & Predominio de cefalea, depresión y fragmentación del sueño \\
\hline $\begin{array}{l}\text { Larsson et al. }{ }^{35} \\
2003\end{array}$ & $2.299 \mathrm{~F}-2.349 \mathrm{M}$ & $\begin{array}{l}\text { Predominio de somnolencia diurna excesiva } \\
\text { Menor reporte de ronquido y apneas presenciadas comparado con los varones }\end{array}$ \\
\hline $\begin{array}{l}\text { Quintana-Gallego et al. }{ }^{5} \\
2004\end{array}$ & $196 \mathrm{~F}-970 \mathrm{M}$ & $\begin{array}{l}\text { Predominio de fatiga, cefalea matinal, insomnio, depresión y uso de sedantes } \\
\text { Menor reporte de apneas presenciadas comparado con los varones }\end{array}$ \\
\hline $\begin{array}{l}\text { Baldwin et al. }{ }^{37} \\
2004\end{array}$ & $3.349 \mathrm{~F}-3.091 \mathrm{M}$ & $\begin{array}{l}\text { Mayor reporte de pobre calidad del sueño, insomnio de conciliación y man- } \\
\text { tención }\end{array}$ \\
\hline $\begin{array}{l}\text { Shepertycky et al. }{ }^{12} \\
2005\end{array}$ & $130 \mathrm{~F}-130 \mathrm{M}$ & Mayor reporte de insomnio, depresión y enfermedad del tiroides \\
\hline $\begin{array}{l}\text { Valipour et al. }{ }^{33} \\
2007\end{array}$ & $782 \mathrm{~F}-2.379 \mathrm{M}$ & Mayor reporte de insomnio, depresión y piernas inquietas \\
\hline $\begin{array}{l}\text { Wahner-Roedler et al. }{ }^{10} \\
2007\end{array}$ & $139 \mathrm{~F}-267 \mathrm{M}$ & $\begin{array}{l}\text { Mayor reporte de falta de energía, insomnio de conciliación, sudoración } \\
\text { nocturna }\end{array}$ \\
\hline $\begin{array}{l}\text { Alotair et al. }{ }^{42} \\
2008\end{array}$ & $191 F-193 M$ & $\begin{array}{l}\text { Mayor reporte de comorbilidades, tales como hipertensión, diabetes, hipoti- } \\
\text { roidismo, asma bronquial e insomnio }\end{array}$ \\
\hline
\end{tabular}

Nota: F: Femenino; M: Masculino. 
En nuestro estudio, el reporte de somnolencia diurna excesiva, sueño poco reparador y la puntuación elevada en la escala de somnolencia de Epworth fueron más prevalentes en las mujeres comparado con los varones pareados por edad y gravedad del trastorno respiratorio del sueño. Aún no está claro si existen diferencias de género en el reporte de somnolencia diurna excesiva en pacientes con trastornos respiratorios del sueño. La correlación positiva entre el IAH y la puntuación de la escala de somnolencia de Epworth fue similar en ambos sexos en el Sleep Heart Health Study $y^{34}$. En otro estudio, Larsson y cols. encontraron que las mujeres roncadoras reportaron más somnolencia durante el día que los hombres ${ }^{35}$. En el Wisconsin Sleep Cohort Study, las mujeres reportaron somnolencia diurna excesiva en mayor proporción comparado con los hombres, incluidas aquellas con y sin criterios diagnósticos de SAHOS ${ }^{1}$. Similar a lo descrito en nuestro estudio, Chervin y cols. encontraron que el género masculino predecía consistentemente una puntuación menor en dos puntos en la escala de somnolencia de Epworth, incluso después de controlar por la edad y gravedad del SAHOS ${ }^{36}$. Se ha planteado que las mujeres reportarían la somnolencia con otros términos menos específicos tales como fatiga, cansancio o falta de energía, lo cual dificultaría el diagnóstico de $\mathrm{SAHOS}^{37}$.

El desempeño de los cuestionarios de sueño fue similar en ambos sexos, siendo discretamente superior el valor predictivo del índice de Flemons y cuestionario STOP-Bang en la pesquisa de SAHOS en las mujeres, mientras que la escala de somnolencia de Epworth mantuvo valor predictivo solo en varones. En ambos sexos, los cuestionarios de sueño STOP y STOP-Bang fueron más sensibles y el índice de Flemons fue más específico en la pesquisa de pacientes con SAHOS moderado-grave ${ }^{7}$.

Las principales características clínicas del SAHOS en las mujeres son el aumento progresivo de la prevalencia entre la cuarta y séptima década de la vida, especialmente después de la menopausia, tienden a tener mayor obesidad, comorbilidades y menor gravedad de la condición comparado con los varones, suelen consultar por síntomas inespecíficos, tales como ansiedad, depresión, fatigabilidad e insomnio, lo cual dificultaría y retrasaría el proceso diagnóstico de esta condición en los servicios de atención primaria.

\section{Referencias}

1. Young T, Palta M, Dempsey J, Skatrud J, Weber S, Badr $\mathrm{S}$. The occurrence of sleep-disordered breathing among middle-aged adults. N Engl J Med 1993; 328: 1230-35.

2. Young T, Hutton R, Finn L, Badr S, Palta M. The gender bias in sleep apnea diagnosis. Are women missed because they have different symptoms? Arch Intern Med 1996; 156: 2445-51.

3. Redline S, Kump K, Tishler PV, Browner I, Ferrette V. Gender differences in sleep disordered breathing in a community-based sample. Am J Respir CritCare Med 1994; 149: 722-6.

4. Bixler EO, Vgontzas AN, Lin HM, Ten Have T, Rein J, Vela-Bueno A, et al. Prevalence of sleep-disordered breathing in women: effects of gender. Am J Respir Crit Care Med 2001; 163: 608-13.

5. Quintana-Gallego E, Carmona-Bernal C, Capote F, Sanchez-Armengol A, Botebol-Benhamou G, Polo-Padillo $\mathrm{J}$, et al. Gender differences in obstructive sleep apnea syndrome: a clinical study of 1166 patients. Respir Med 2004; 98: 984-9.

6. Peppard PE, Young T, Barnet JH, Palta M, Hagen EW, Hla KM. Increased prevalence of sleep-disordered breathing in adults. Am J Epidemiol 2013; 177: 1006-14.

7. Saldías F, Gassmann J, Canelo A, Uribe J, Díaz O. Evaluación de los cuestionarios de sueño en la pesquisa de pacientes con síndrome de apneas obstructivas del sueño. Rev Med Chile 2018; 146: 1123-34.

8. Franklin KA, Lindberg E. Obstructive sleep apnea is a common disorder in the population-a review on the epidemiology of sleep apnea. J Thorac Dis 2015; 7: 131122.

9. Pillar G, Lavie P. Psychiatric symptoms in sleep apnea syndrome: effects of gender and respiratory disturbance index. Chest 1998; 114: 697-703.

10. Wahner-Roedler DL, Olson EJ, Narayanan S, Sood R, Hanson AC, Loehrer LL, et al. Gender-specific differences in a patient population with obstructive sleep apnea-hypopnea syndrome. Gend Med 2007; 4: 329-38.

11. Ye L, Pien GW, Weaver TE. Gender differences in the clinical manifestation of obstructive sleep apnea. Sleep Med 2009; 10: 1075-84.

12. Shepertycky MR, Banno K, Kryger MH. Differences between men and women in the clinical presentation of patients diagnosed with obstructive sleep apnea syndrome. Sleep 2005; 28: 309-14.

13. Kapur V, Strohl KP, Redline S, Iber C, O’Connor G, Nieto J. Underdiagnosis of sleep apnea syndrome in U.S. communities. Sleep Breath 2002; 6: 49-54.

14. Ware JC, McBrayer RH, Scott JA. Influence of sex and 
age on duration and frequency of sleep apnea events. Sleep 2000; 23: 165-70.

15. Lin CM, Davidson TM, Ancoli-Israel S. Gender differences in obstructive sleep apnea and treatment implications. Sleep Med Rev 2008; 12: 481-96.

16. Krishnan V, Collop NA. Gender differences in sleep disorders. Curr Opin Pulm Med 12: 383-9.

17. Netzer NC, Stoohs RA, Netzer CM, Clark K, Strohl KP. Using the Berlin Questionnaire to identify patients at risk for the sleep apnea syndrome. Ann Intern Med 1999; 131: 485-91.

18. Buysse DJ, Reynolds CF, Monk TH, Berman SR, Kupfer DJ. The Pittsburgh Sleep Quality Index: A new instrument for psychiatric practice and research. Psychiatry Res 1989; 28: 193-213.

19. Chung F, Yegneswaran B, Liao P, Chung SA, Vairavanathan S, Islam S. STOP questionnaire: a tool to screen patients for obstructive sleep apnea. Anesthesiology 2008; 108: 812-21.

20. Chung F, Abdullah HR, Liao P. STOP-Bang Questionnaire: A practical approach to screen for obstructive sleep apnea. Chest 2016; 149: 631-8.

21. Flemons WW, Whitelaw WA, Brant R, Remmers JE. Likelihood ratios for a sleep apnea clinical prediction rule. Am J Respir Crit Care Med 1994; 150: 1279-85.

22. Johns MW. A new method for measuring daytime sleepiness: the Epworth sleepiness scale. Sleep 1991; 14: 540-5.

23. Beck AT, Steer RA, Brown GK. BDI-II. Manual for the Beck Depression Inventory-Second Edition. San Antonio, TX: Psychological Corporation; 1996.

24. Zigmond AS, Snaith RP. The Hospital Anxiety and Depression Scale. Acta Psychiatr Scand 1983;67:361-70.

25. Nagappa M, Liao P, Wong J, Auckley D, Ramachandran SK, Memtsoudis S, et al. Validation of the STOP-Bang questionnaire as a screening tool for obstructive sleep apnea among different populations: A systematic review and meta-analysis. PLoS One 2015; 10: e0143697.

26. Rechtschaffen A, Kales A. A Manual for the standardized terminology, techniques and scoring system for sleep stages of human subjects. Washington DC: US Government Printing Office; NIH Publication 204; 1968.

27. Kapur VK, Auckley DH, Chowdhuri S, Kuhlmann DC, Mehra R, Ramar K, et al. Clinical Practice Guideline for Diagnostic Testing for Adult Obstructive Sleep Apnea:
An American Academy of Sleep Medicine Clinical Practice Guideline. J Clin Sleep Med 2017; 13: 479-504.

28. Ambrogetti A, Olson LG, Saunders NA. Differences in the symptoms of men and women with obstructive sleep apnoea. Aust N Z J Med 1991; 21: 863-6.

29. O'Connor C, Thornley KS, Hanly PJ. Gender differences in the polysomnographic features of obstructive sleep apnea. Am J Respir Crit Care Med 2000; 161: 1465-72.

30. Valipour A, Lothaller H, Rauscher H, Zwick H, Burghuber OC, Lavie P. Gender-related differences in symptoms of patients with suspected breathing disorders in sleep: a clinical population study using the sleep disorders questionnaire. Sleep 2007; 30: 312-9.

31. Gottlieb DJ, Whitney CW, Bonekat WH, Iber C, James GD, Lebowitz M, et al. Relation of sleepiness to respiratory disturbance index: the Sleep Heart Health Study. Am J Respir Crit Care Med 1999; 159 (2): 502-7.

32. Larsson LG, Lindberg A, Franklin KA, Lundback B. Gender differences in symptoms related to sleep apnea in a general population and in relation to referral to sleep clinic. Chest 2003; 124: 204-11.

33. Chervin RD, Aldrich MS. The Epworth sleepiness scale may not reflect objective measures of sleepiness or sleep apnea. Neurology 1999; 52: 125-31.

34. Baldwin CM, Kapur VK, Holberg CJ, Rosen C, Nieto FJ, Sleep Heart Health Study Group. Associations between gender and measures of daytime somnolence in the Sleep Heart Health Study. Sleep 2004; 27: 305-11.

35. Guilleminault C, Stoohs R, Kim YD, Chervin R, Black J, Clerk A. Upper-airway sleep-disordered breathing in women. Ann Intern Med 1995; 122: 493-501.

36. Chervin RD. Sleepiness, fatigue, tiredness, and lack of energy in obstructive sleep apnea. Chest 2000; 118 : 372-9.

37. Walker RP, Durazo-Arvizu R, Wachter B, Gopalsami C. Preoperative differences between male and female patients with sleep apnea. Laryngoscope 2001; 111: 1501-5.

38. Resta O, Caratozzolo G, Pannacciulli N, Stefano A, Giliberti T, Carpagnano GE, et al. Gender, age and menopause effects on the prevalence and the characteristics of obstructive sleep apnea in obesity. Eur J Clin Invest 2003; 33: 1084-9.

39. Alotair H, Bahammam A. Gender differences in Saudi patients with obstructive sleep apnea. Sleep Breath 2008; 12: 323-9. 